\title{
ANATOMIA COMPARATIVA DA MADEIRA DE QUATRO ESPÉCIES DA SUBTRIBO BACCHARINAE LESSING (ASTERACEAE - ASTEREAE) ${ }^{1}$
}

\author{
ANABELA SILVEIRA DE OLIVEIRA² JOSÉ NEWTON CARDOSO MARCHIORI ${ }^{3}$
}

\section{RESUMO}

O presente trabalho trata do estudo anatômico das madeiras de Heterothalamus alienus (Sprengel) O. Kuntze, Heterothalamus psiadioides Lessing, Heterothalamus rupestris Deble, Oliveira \& Marchiori e Heterothalamulopis wagenitzii (Hellwig) Deble, Oliveira \& Marchiori. Nas quatro espécies foram observados vasos tipicamente pequenos e em padrão dendrítico, placas de perfuração simples e parênquima paratraqueal. A presença de estratificação e de espessamentos espiralados, assim como a abundância de parênquima axial e a morfologia das células de raio, serviram para a separação das espécies, em chave dicotômica.

Palavras chave: Heterothalamus, Heterothalamulopsis, Asteraceae, Baccharinae, Anatomia da Madeira.

\section{ABSTRACT}

The present work deals with the wood anatomy of Heterothalamus alienus (Sprengel) O. Kuntze, Heterothalamus psiadioides Lessing, Heterothalamus rupestris Deble, Oliveira \& Marchiori and Heterothalamulopis wagenitzii (Hellwig) Deble, Oliveira \& Marchiori. The four species showed small vessels in dendritic pattern, only simple perforation plates and paratraqueal parenchyma. The presence of storied structure, and spiral thickenings in vessel walls, as well as the abundance of axial parenchyma and the morphology of radial cells, served to set apart the distinct species in a dichotomous key.

Key words: Heterothalamus, Heterothalamulopsis, Asteraceae, Baccharinae, Wood Anatomy.

\section{INTRODUÇÃO}

Com cerca de 1.100 gêneros e 25.000 espécies, as Asteraceae distribuem-se pelas regiões tropicais, subtropicais e temperadas do mundo, estando bem representadas em todos os tipos de habitat, com exceção do aquático. No Brasil, a família compreende cerca de 180 gêneros (Barroso, 1991).

Os gêneros americanos da subtribo Baccharinae Less. (tribo Astereae Cassini) caracterizam-se pela presença de flores hermafroditas, funcionalmente masculinas por aborto do gineceu. No Brasil, o grupo está representado por 4 gêneros: Baccharidastrum Cabrera, Baccharis Linnaeus, Heterothalamus
Lessing e Heterothalamulopsis Deble, Oliveira \& Marchiori (Deble, Oliveira \& Marchiori, 2005).

Composto de três espécies, o gênero Heterothalamus Lessing tem distribuição geográfica limitada ao sul do Brasil (Santa Catarina e Rio Grande do Sul), Uruguai e centro da Argentina (províncias de Córdoba, San Luis e La Rioja).

O gênero Heterothalamulopsis Deble, Oliveira \& Marchiori compreende uma única espécie, restrita à borda dos "Aparados da Serra", no Rio Grande do Sul (Cambará do Sul, São José dos Ausentes) e Santa Catarina (municípios de Bom Jardim da Serra, Bom Retiro, Grão Pará, São Joaquim e Urubici).

\footnotetext{
Artigo recebido em 03/03/2005 e aceito para publicação em 07/07/2005.

2 Bióloga, MSc., bolsista Capes, doutoranda do Programa de Pós-graduação em Engenharia Florestal, Centro de Ciências Rurais, Universidade Federal de Santa Maria, CEP 97105-900, Santa Maria (RS). anabela.biol@mail.ufsm.br

3 Engenheiro Florestal, bolsista de Produtividade em Pesquisa do CNPq, Dr., Professor Titular do Departamento de Ciências Florestais, Centro de Ciências Rurais, Universidade Federal de Santa Maria, CEP 97105-900, Santa Maria (RS).balduinia@mail.ufsm.br
} 
O xilema secundário das Asteraceae é marcado por anéis de crescimento pouco evidentes, pela porosidade difusa ou semidifusa e por vasos tipicamente pequenos, ocasionalmente de tamanho médio, com placas de perfuração simples, mais raramente escalariforme-irregulares ou reticuladas. Providos de pontoações intervasculares alternas, diminutas até moderadamente grandes, os vasos reúnem-se em agrupamentos diagonais e racemiformes irregulares, compondo, por vezes, um nítido padrão dendrítico. O parênquima axial, tipicamente paratraqueal e seriado, pode ser estratificado ou composto de células fusiformes. Os raios, tipicamente com 4-10 células de largura e escassos unisseriados de baixa altura, são exclusivamente unisseriados em poucas espécies e, por vezes, estratificados; os multisseriados, reúnem uma mistura irregular de células eretas e procumbentes. As fibras, pequenas e de pontoações simples, ocasionalmente septadas, variam de comprimento médio até muito curtas. Tilos e traqueídeos vasicêntricos, por sua vez, são aspectos pouco freqüentes, bem como a presença de canais intercelulares em raios. Com relação a cristais, não constam referências do caráter na família (Metcalfe \& Chalk, 1972; Record \& Hess, 1949)

\section{MATERIAL E MÉTODOS}

As amostras de madeira utilizadas na pesquisa procedem de dois pontos de coleta: na Serra do Sudeste gaúcho, entre os municípios de Caçapava do Sul e Santana da Boa Vista, e nos Aparados da Serra Geral, em Cambará do Sul.

Para o estudo anatômico, foram selecionados três indivíduos adultos de cada uma das espécies de Heterothalamus; com relação a Heterothalamulopsis wagenitzii, coletou-se uma única amostra, devido a sua raridade. Dos indivíduos escolhidos, foram extraídos três discos do tronco, com espessura aproximada de $2,5 \mathrm{~cm}$, além de material botânico, com vistas à confec- ção de exsicatas, incorporadas ao Herbário do Departamento de Ciências Florestais da Universidade Federal de Santa Maria.

Das amostras de madeira foram preparados corpos-de-prova, tomados em região de alburno e orientados para a obtenção de cortes anatômicos nos planos transversal, longitudinal radial e longitudinal tangencial.

A microtomia, realizada no Laboratório de Anatomia da Madeira da Universidade Federal do Paraná, seguiu procedimentos usuais naquela instituição: os corpos-de-prova foram amolecidos por fervura em água e seccionados em micrótomo de deslizamento, com espessura nominal de 18 micrômetros. Usou-se tripla coloração, com acridina-vermelha, crisoidina e azulde-astra (Dujardin, 1964), seguida de desidratação em série alcoólica e montagem de lâminas permanentes, com "Entellan".

Para as lâminas de macerado, seguiu-se o método de Jeffrey (Freund, 1970), usando-se coloração com safranina e montagem permanente, com "Entellan".

A terminologia, número de medições e descrição da estrutura anatômica seguiram as recomendações da Copant (1973). Para a determinação da percentagem dos diferentes tipos celulares, seguiu-se a metodologia recomendada por Marchiori (1980). As medições foram realizadas em microscópico Carl Zeiss, no Laboratório de Anatomia da Madeira da Universidade Federal de Santa Maria. Os dados quantitativos, reunidos em tabelas, bem como os números de registro do material coletado, foram publicados juntamente com as descrições anatômicas das distintas espécies (Oliveira, Deble, Marchiori \& Denardi, 2005; Oliveira, Marchiori \& Deble, 2005; Oliveira \& Marchiori, 2005). Com relação aos dados quantitativos da estrutura anatômica, eles serviram de base para a elaboração dos gráficos e da chave dicotômica que, no presente trabalho, buscam contribuir para o conhecimento anatômico da subtribo Baccharinae Lessing. 


\section{ANÁLISE ESTRUTURAL DO XILEMA}

Vasos:

De modo geral, os detalhes anatômicos de vasos coincidem com as referências de Metcalfe \& Chalk (1972), para a família, e com o descrito para a tribo Astereae, por Carlquist (1960).

Os elementos vasculares fornecem poucos caracteres de valor diagnóstico para separação de espécies. Placas de perfuração simples e pontoações em arranjo alterno, por exemplo, são comuns às quatro espécies estudadas.

O volume ocupado pelos vasos, por sua vez, varia desde $13 \%$, em Heterothalamus psiadioides, até $16 \%$ e $19 \%$, em Heterothalamus rupestris e Heterothalamus alienus, respectivamente. Para Heterothalamulopsis wagenitzii, a percentagem de vasos é ainda menor, correspondendo a $12 \%$ do volume da madeira (Figura 1).

As quatro espécies apresentam vasos extremamente numerosos e em distribuição difusa, não uniforme, dispostos em agrupamentos diagonais e racemiformes, com raros poros solitários.

Quanto ao diâmetro, os vasos variam de extremamente pequenos a pequenos. Em Heterothalamus alienus e $H$. rupestris, a seção é predominantemente poligonal, diferindo, neste aspecto, de $H$. psiadioides e Heterothalamu- lopsis wagenitzii, que apresentam vasos de seção poligonal até oval. Na figura 2, vê-se que as três espécies de Heterothalamus apresentam poros nitidamente maiores do que Heterothalamulopsis wagenitzii.

A freqüência de poros $/ \mathrm{mm}^{2}$ proporciona poucos subsídios para separação das espécies (Figura 3). Em Heterothalamus alienus, todavia, este índice é mais elevado, permitindo sua separação de $H$. rupestris e $H$. psiadioides. Apesar da pequena variação no comprimento dos elementos vasculares (Figura 4), Heterothalamus psiadioides distingue-se por tê-los mais longos do que $H$. alienus e $H$. rupestris. De acordo com Carlquist (1977), elementos vasculares curtos indicam maior especialização do que elementos longos, dentro de um mesmo gênero botânico.

Parênquima axial:

A análise do parênquima axial fornece importantes subsídios para a identificação das espécies em estudo (Figura 5).

A percentagem do tecido ultrapassa a $25 \%$ do volume da madeira em Heterothalamus alienus, ficando pouco abaixo de $22 \%$ em Heterothalamus rupestris e $H$. psiadioides, e entre 10 e $16 \%$ em Heterothalamulopsis wagenitzii.

\section{$(\%)$}

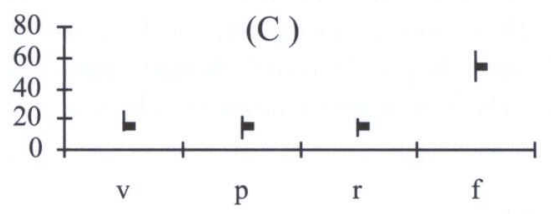

$(\%)$

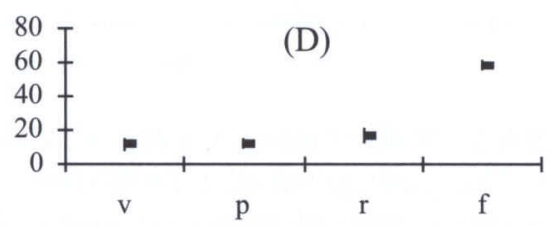

FIGURA 1 - Percentagem dos tecidos constituintes da madeira, em Heterothalamus alienus (A), H. psiadioides (B), H. rupestris (C) e Heterothalamulopsis wagenitzii (D). ( $\mathrm{v}=$ vasos; $\mathrm{p}=$ parênquima; $\mathrm{r}=\mathrm{raios} ; \mathrm{f}=$ fibras). 


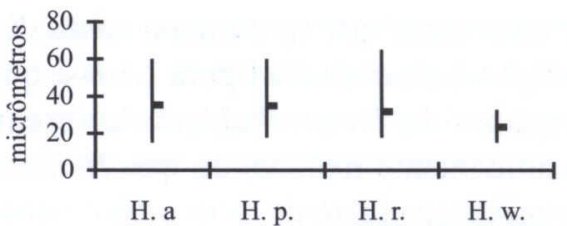

FIGURA 2 - Valores mínimo, médio e máximo do diâmetro de poros, em Heterothalamus alienus (H.a.), Heterothalamus psiadioides (H.p.), Heterothalamus rupestris (H.r.) e Heterothalamulopsis wagenitzii (H.w.).

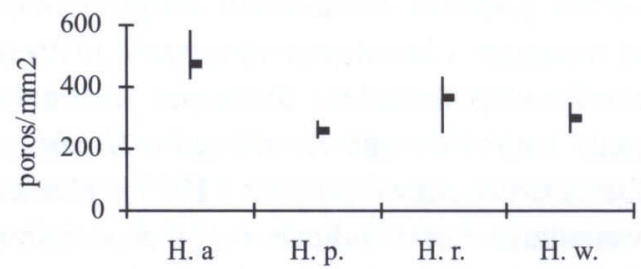

FIGURA 3 - Valores mínimo, médio e máximo da frequiência de poros $/ \mathrm{mm}^{2}$, em Heterothalamus alienus (H.a.), Heterothalamus psiadioides (H.p.), Heterothalamus rupestris (H.r.) e Heterothalamulopsis wagenitzii (H.w.).

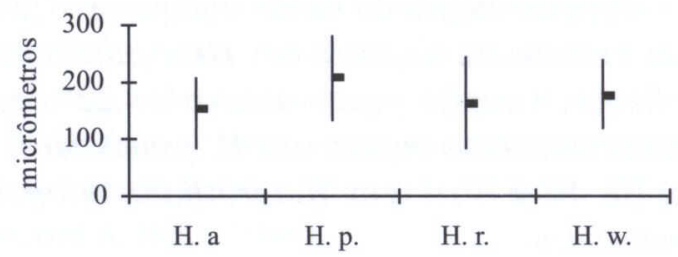

FIGURA 4 - Valores mínimo, médio e máximo do comprimento de elementos vasculares, em Heterothalamus alienus (H.a.), Heterothalamus psiadioides (H.p.), Heterothalamus rupestris (H.r.) e Heterothalamulopsis wagenitzii (H.w.).

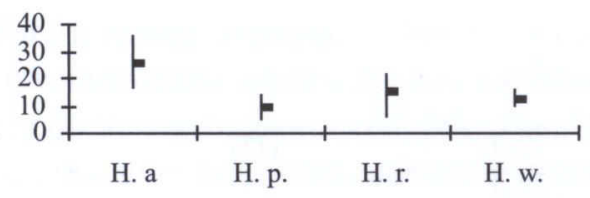

FIGURA 5 - Valores mínimo, médio e máximo da fração (\%) de parênquima axial, em Heterothalamus alienus (H.a.), Heterothalamus psiadioides (H.p.), Heterothalamus rupestris (H.r.) e Heterothalamulopsis wagenitzii (H.w.).

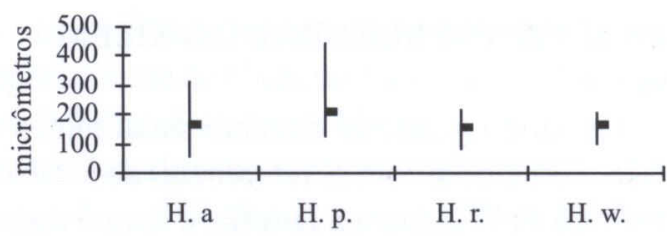

FIGURA 6 - Valores mínimo, médio e máximo da altura das séries parenquimáticas $(\mu \mathrm{m})$, em Heterothalamus alienus (H.a.), Heterothalamus psiadioides (H.p.), Heterothalamus rupestris (H.r.) e Heterothalamulopsis wagenitzii (H.w.).

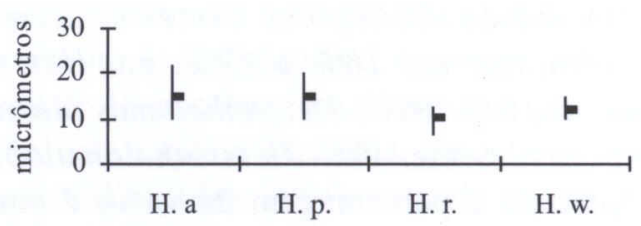

FIGURA 7 - Valores mínimo, médio e máximo da largura de células fusiformes $(\mu \mathrm{m})$, em Heterothalamus alienus (H.a.), Heterothalamus psiadioides (H.p.), Heterothalamus rupestris (H.r.) e Heterothalamulopsis wagenitzii (H.w.).

O parênquima, tipicamente paratraqueal nas quatro espécies, varia de escasso a vasicêntrico em Heterothalamus psiadioides, $H$. rupestris e Heterothalamulopsis wagenitzii; Heterothalamus alienus, ao contrário, distingue-se pelo abundante parênquima estratificado.

As séries parenquimáticas variam quanto à altura em micrômetros, permitindo a separação de Heterothalamus psiadioides das demais espécies, com base nesse caráter (Figura 6). Heterothalamus rupestris, por sua vez, pode ser identificada pelas células fusiformes mais estreitas (Figura 7).

O número de células por série mostra pouca variação nas espécies em estudo. No caso de Heterothalamus alienus, encontram-se duas, raramente 3 ou 4 células; em Heterothalamus rupestris, elas são sempre em número de duas, ao passo que em Heterothalamus psiadioides e Heterothalamulopis wagenitzii, as séries parenquimáticas reúnem duas, raro três células. 


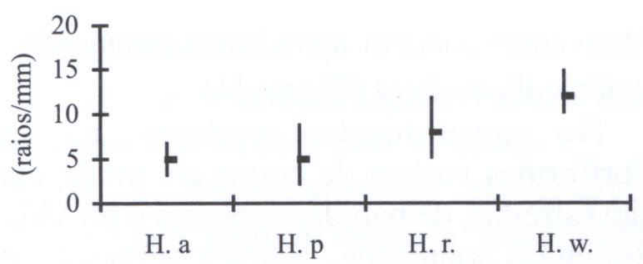

FIGURA 8 - Valores mínimo, médio e máximo da freqüência de raios, em Heterothalamus alienus (H.a.), Heterothalamus psiadioides (H.p.), Heterothalamus rupestris (H.r.) e Heterothalamulopsis wagenitzii (H.w.).

\section{Raios:}

O volume ocupado pelo tecido radial mostra pouca variação entre as espécies de Heterothalamus, oscilando de 9\% (Heterothalamus psiadioides) a $15 \%$ (Heterothalamus rupestris). No caso de Heterothalamulopsis wagenitzii, esse valor é ainda maior, ficando em torno de $17 \%$ (Figura 1).

A estrutura radial caracteriza-se pela heterogeneidade, reunindo células procumbentes e quadradas (Tipo III), nas três espécies de Heterothalamus, e células procumbentes, quadradas até distintamente eretas (Tipo II), em Heterothalamulopsis wagenitzii. $\mathrm{O}$ caráter reveste-se de importância para a identificação anatômica, permitindo, inclusive, a separação

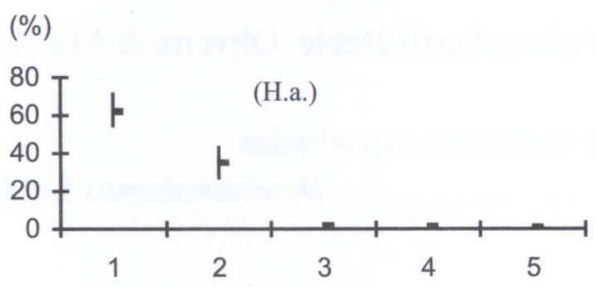

(\%)

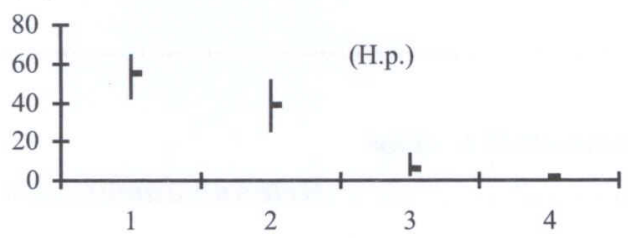

dos dois gêneros em estudo na subtribo Baccharinae; a esse respeito, resta lembrar que entre as espécies de Baccharis, de acordo com Carlquist (1966), os raios compõem-se de células quadradas, de células procumbentes, quadradas e eretas, ou de uma mistura destes tipos.

A figura 8 mostra a distribuição da frequiência de raios para as quatro madeiras em estudo. Nas três espécies de Heterothalamus, os raios variam de muito pouco frequientes a muito numerosos, permitindo fácil separação de Heterothalamulopsis wagenitzii, cujos raios são invariavelmente muito numerosos.

A percentagem das classes de raios quanto à largura em número de células (Figura 9) também fornece importantes subsídios para a identificação. Em Heterothalamus alienus e $H$. rupestris predominam raios uni, bi e trisseriados. Em Heterothalamus psiadioides, o tecido radial compõe-se, em sua maioria, de raios uni e bisseriados, havendo poucos trisseriados e raros tetrasseriados. Em Heterothalamulopsis wagenitzii, por sua vez, embora também predominem os uni e bisseriados, a presença de raios com até cinco células de largura permite sua fácil separação do outro gênero em estudo.

A altura dos raios unisseriados em micrômetros (Figura 10) e em número de células permite se-

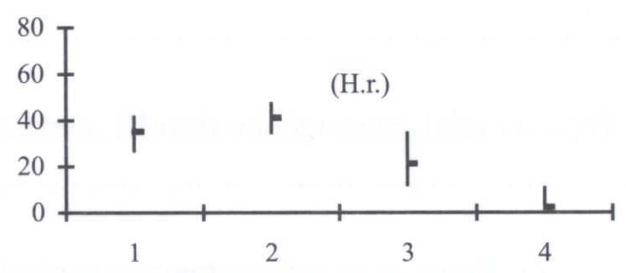

(\%)

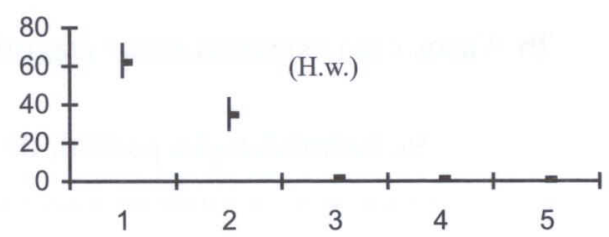

FIGURA 9 - Percentagem das classes de raios quanto à largura em número de células, para as quatro espécies estudadas ( $1=$ unisseriados, $2=$ bisseriados, $3=$ trisseriados, $4=$ tetrasseriados, $5=$ pentasseriados $)$. 


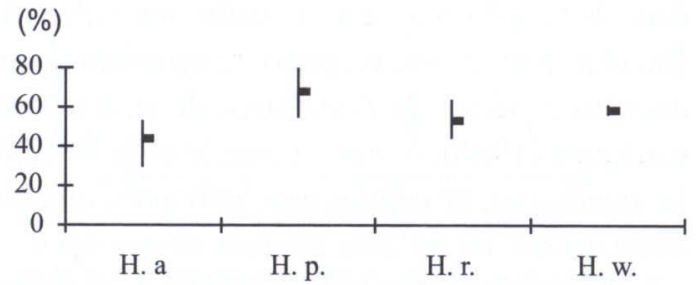

FIGURA 11 - Percentagem de fibras libriformes, em Heterothalamus alienus (H.a.), Heterothalamus psiadioides (H.p.), Heterothalamus rupestris (H.r.) e Heterothalamulopsis wagenitzii (H.w.).

gregar Heterothalamus alienus das outras em estudo, por ter raios muito baixos, com 1-4-8 células de altura. Nas demais espécies, os raios variam de muito baixos até medianos, com 19 a 36 células de altura máxima.

Fibras:

$\mathrm{O}$ volume percentual de fibras exibe variação significativa nas quatro espécies. Heterothalamus psiadioides separa-se pela alta percentagem de fibras (superior a 75\%), ao passo que em H. alienus este valor fica abaixo de 55\%. Em Heterothalamulopsis wagenitzii, por sua vez, as fibras correspondem, aproximadamente, a $60 \%$ do volume da madeira (Figura 11).

No conjunto das espécies, as fibras libriformes variam de curtas até muito curtas, são estreitas, de paredes espessas e providas de diminutas pontoações simples; resta salientar a ausência de septos nas mesmas.

\section{Outros caracteres:}

Não foram observados canais secretores, tubos lactíferos e taniníferos, líber incluso e máculas medulares, nas quatro espécies em estudo. Os anéis de crescimento são também indistintos, nas quatro espécies. A estratificação parcial de vasos e parênquima axial, restrita a Heterothalamus alienus, confere caráter relativamente evoluído ao xilema dessa espécie, de acordo com Metcalfe \& Chalk (1972) e Record \& Hess (1949).

\section{CHAVE PARA IDENTIFICAÇÃO ANATÔ- MICA DAS ESPÉCIES}

A análise do material em estudo permite a separação das espécies pela seguinte chave dicotômica, com base na presença de espessamentos espiralados em vasos, na abundância de parênquima axial e na estrutura do tecido radial:

1a. Tecido radial heterogêneo tipo II, com células procumbentes, quadradas até distintamente eretas

Heterothalamulopsis Deble, Oliveira \& Marchiori

1b. Tecido radial heterogêneo tipo III, com células procumbentes e quadradas Heterothalamus Lessing

2a. Vasos sem espessamentos espiralados

Heterothalamus rupestris

2b. Vasos com espessamentos espiralados.

3a. Estratificação parcial, de parênquima axial e vasos

Heterothalamus alienus

3b. Parênquima axial e vasos, não estratificados 
BIBLIOGRAFIA

Barroso, G. M. Sistemática de angiospermas do Brasil. Viçosa: Editora UFV, 1991. v. 3, 326p.

Carlquist, S. Wood anatomy of Astereae (Compositae). Tropical Woods, n. 113, p. 54-84, 1960.

Carlquist, S. Wood anatomy of Compositae: a summary, with comments on factors controlling wood evolution. Aliso, v. 6, n. 2, p. 25-44, 1966.

Carlquist, S. Ecological factors in wood evolution: a floristic approach. American Journal of Botany, v. 64, n.7, p. 887-896, 1977.

Copant - Comissão Panamericana de Normas Técnicas. Descrição macroscópica, microscópica e geral da madeira - esquema I de recomendação. Colômbia, 19p. 1973 (COPANT 30).

Deble, L. P., Oliveira, A. S., Marchiori, J. N. C. O gênero Heterothalamus Lessing e táxones afins. Balduinia, Santa Maria, n.1, p.1-20, 2005.

Dujardin, E. P. Eine neue Holz-Zellulosenfaerbung. Mikrokosmos, n. 53, p. 94, 1964.

Freund, H. Handbuch der Mikroskopie in der Technik. Frankfurt: Umschan Verlag, 1970. 379p. Iawa. List of microscopic features for hardwood identification. IAWA Bulletin, v. 10, n. 3, p. 219-332, 1989.
Marchiori, J. N. C. Estudo anatômico do xilema secundário de algumas espécies dos gêneros Acacia e Mimosa, nativas no Estado do Rio Grande do Sul. Curitiba: UFPR, 1980, 186p. Dissertação (Mestrado em Engenharia Florestal) - Universidade Federal do Paraná, Curitiba.

Marchiori, J. N. C., Brum E. T. Anatomia da madeira do guamirim-facho, Calyptranthes concina DC. Ciência Rural, Santa Maria, v. 27, n. 2, p. 217 222, 1997.

Metcalfe, C. R., Chalk, L. Anatomy of the Dicotyledons. Oxford: Clarendon Press, 1972. v. 2, 1500p.

Oliveira, A. S. de, Marchiori, J.N.C., Deble, L. P. Anatomia do lenho de Heterothalamulopsis wagenitzii (F. Hellw.) Deble, Oliveira \& Marchiori (Astereae - Asteraceae). Balduinia, Santa Maria, n. 2, p. 13-18, 2005.

Oliveira, A. S. de, Marchiori, J. N. C. Anatomia do lenho de Heterothalamus psiadioides Lessing (Asteraceae-Astereae). Balduinia, Santa Maria, n. 4, p. 20-24, 2005.

Oliveira, A. S. de, Deble, L. P., Marchiori, J. N. C., Denardi, L. Anatomia da madeira de duas espécies do gênero Heterothalamus Lessing (Asteraceae), nativas no Rio Grande do Sul. Ciência Florestal, Santa Maria, v. 15, n. 1, p. 9-19, 2005.

Record, S. J., Hess, R. W. Timbers of The New World. New Haven: Yale University Press, 1949. 640p 